\title{
Accessibility of Medical Facilities in Multiple Traffic Modes: A Study in Guangzhou, China
}

\author{
Juhua Wu, ${ }^{1}$ Zeqian Cai, ${ }^{1}$ and Honglei Li $\mathbb{D}^{2}$ \\ ${ }^{1}$ Guangdong University of Technology, Yinglong Road 161, Guangzhou 510006, China \\ ${ }^{2}$ School of Management, Liaoning Normal University, 850 Huanghe Road, Dalian 116029, China \\ Correspondence should be addressed to Honglei Li; lhl@lnnu.edu.cn
}

Received 10 August 2020; Revised 10 September 2020; Accepted 11 September 2020; Published 24 September 2020

Academic Editor: Jun Yang

Copyright (c) 2020 Juhua Wu et al. This is an open access article distributed under the Creative Commons Attribution License, which permits unrestricted use, distribution, and reproduction in any medium, provided the original work is properly cited.

One of the major contradictions in China's healthcare service is the serious imbalance between the supply and demand of medical resources and the uneven distribution of medical resources. Although the government intended to provide equal access for all, in the final evaluation, fairness of the distribution of services was usually dictated by location. Measuring and predicting access to social services can help to adjust and better accommodate underserved regions. 3SFCA modes based on the Gaussian function are proposed to delineate the service area of providers delivering social services and produce a probability metric that maps the equity of the medical services in multiple traffic modes. We identified the types of medical shortage areas and analyzed the distribution characteristics of medical accessibility in Guangzhou. The results showed that the accessibility of medical facilities in the multiple traffic modes varied greatly. The accessibility level of medical service resources was the highest in the driving mode, followed by the public traffic mode and bike-riding mode. The walking mode had the lowest accessibility. Finally, the paper provided advice for further improvement of the equal distribution of medical service resources in Guangzhou.

\section{Introduction}

It was reported that China's Healthcare Access and Quality (HAQ) Index ranked in top 50 of 195 countries and territories, which ranked the third in the advancement list [1]. However, with the rapid growth of Chinese economy and the living standard, the demand for better medical service bursts out in China, which leads to the insufficiency of urban medical facilities.

At present, even in the same city, the public health and medical service is still unevenly developed in different regions, which causes the most serious contradiction in China's public health and medical service domain. For example, Chen and Yeh stated that in Guangzhou, the third developed city of China, the low-income population is mainly located in the suburban districts while the medical facilities are located in the center of the city, which causes serious accessibility problems [2]. This is only an epitome of the Chinese medical service system.

As for the accessibility of medical facilities, related studies have been launched for years. Hare and Barcus found that people who are within 45-minute distance from hospitals are apt to be socially and economically marginalized while the utility of medical service is higher in the areas with poor communications [3]. Mcgrail and Humphreys evaluated the two-step floating catchment area method (2SFCA) and used dynamic impedance to measure the accessibility of the primary care in the rural areas of Victoria, Australia [4]. Luo and Wang measured the spatial accessibility to healthcare in the Chicago region in a GIS environment and analyzed the sensitivity of the results by using the traffic time threshold range in the FCA method and the travel friction coefficient in the gravity model [5]. Wan et al. proposed a three-step floating catchment area method (3SFCA) for analyzing spatial access to health services to minimize the overestimated demand of medical service [6].

In addition, more studies focused on integrating diverse catchment area modes with floating catchment area methods to explore the difference among barrier-free modes in different spatial scales [7, 8]. Based on 2SFCA, Mao and Nekorchuk proposed a multicatchment area 2SFCA method to measure the spatial accessibility to healthcare for 
populations in Florida [9]. Ma et al. introduced Gaussian weighted algorithm and 3SFCA to explore the hierarchical accessibility of three-level medical facilities in Wuhan, China [10]. Langford et al. improved 2SFCA with road network data to describe how public transport availability and frequency can be incorporated into FCA models to provide a more realistic appraisal of accessibility for those population groups most likely to be reliant on public means of transport to access health care services [11]. Higgs et al. used enhanced 2SFCA techniques to investigate the impact of different modes of travel (car versus bus) on associations between different measures of general practitioner (GP) supply and area level deprivation and the percentage of elderly patients [12].

In recent years, the accessibility of medical facilities has attracted the attention of Chinese researchers. Zhu improved the 2SFCA and inverted 2SFCA to explore the crowdedness and potential accessibility of medical service with the perspective of medical service supply and demand [13]. Chen used the Gaussian 2SFCA method to analyze the spatiotemporal accessibility of medical service for the elderly in downtown Wuhan city [14]. Wang used the improved 2SFCA method to analyze the equality and spatiotemporal layout of medical service in Shanghai [15]. Che used the gravity accessibility model to evaluate the spatial layout of Shanghai's tertiary hospitals [16].

As discussed above, the related study in developed countries started earlier than that in China and initially proposed powerful models or algorithms on accessibility issues. 2-step and 3-step floating catchment area methods are the main technologies for the measurement of accessibility of medical facilities. These models or algorithms have also been upgraded from single index to multiple indexes, and the involved traffic mode is also extended from single mode to multiple modes. With the improvement of models and the extension of traffic modes, it was found that (a) the average accessibility is decreased with the increase of flexibility in multicatchment area mode and (b) compared with the single-catchment area mode, the number of recognized areas with insufficient medical service and the recognized population is also decreased.

Compared with the foreign studies, related studies in China are concentrated on the improvement of current models or algorithms and pay more attention to the data quality, data acquisition, and the difference between the accessibility in urban and suburban areas, while the accessibility of medical facilities in multiple traffic modes has not been widely concerned. Therefore, in this study, Guangzhou as a typical city with multiple traffic resources was taken as the research area. The healthcare accessibility of residents in various regions in different traffic modes (driving mode, public traffic mode, bike-riding mode, and walking mode) was measured with the 3SFCA method. Based on the analysis of the accessibility in different traffic modes, the types and features of regions with insufficient medical facilities were identified. This was helpful to provide solutions to the inequality of medical services. In this study, new ideas for the application and improvement of 3SFCA method were also explored.
This paper is organized as follows.

In Section 1, the importance of accessibility of medical facilities and the related studies are discussed. In Section 2, the data sources are introduced and the methods for accessibility measurement and determination of the size of catchment area are designed. Many models are compared and the 3SFCA method combined with Gaussian function is selected. In Section 3, taking Guangzhou as an example, the healthcare accessibility of residents in various areas in four different traffic modes is measured and discussed. The suggestion for the government to optimize the medical service resource allocation is provided. Section 4 gives the conclusion.

\section{Data and Methods}

2.1. Dataset. Guangzhou is one of biggest cities situated in the southern part of China. It is also the political, economic, and cultural center of South China. The total area of the city is 7434.4 square kilometers, and the resident population of the city was about 14.5 million by the end of 2017. As one of the top 3 medical centers of China, there were 269 hospitals and more than 160 thousand medical professionals by the end of 2019. However, the traffic situation in Guangzhou is also as complicated as other big cities in China, and the regions of the city are also unequally developed. Residents often travel for medical service in many different traffic modes according to the traffic situation and income level. Guangzhou can be taken as a good representative of the public healthcare accessibility study.

In this study, the following related data about the city were collected.

(i) District and population data: district data came from the adjusted administrative division map (2014 version) of Guangzhou where administrative districts existed including Yuexiu, Huizhu, liwan, Tianhe, Baiyun, Huangpu, Huadu, Panyu, Nansha, Conghua, and Zengcheng. The longitude and latitude information of each district administrative center were generated by DataMap [17]. Through matching the geographical location data with the administrative organization list of Guangzhou, 170 streets were selected for investigation. The population data of each street were extracted from the statistics yearbook 2017 in official websites of each district offices.

(ii) Medical facility data: the hospitals' information in this study came from the List of Major Hospitals in Guangzhou published by Guangdong Provincial Government (GDDATA, 2018) [18] and the National Medical Database published by the National Health Commission of the People's Republic of China (NHC 2020) [19]. This study added address, geographic coordinates, and grade features to the facility information in the hospital list. Considering the category and grade, 129 hospitals remained. Among all hospitals, 37 tertiary hospitals, $48 \mathrm{sec}-$ ondary hospitals, 28 primary hospitals, and 16 
unclassified hospitals were taken into account. The unclassified hospitals were hospitals affiliated to organizations such as schools and companies. However, the unclassified hospitals also provided public healthcare for the public.

(iii) Routine data: generally speaking, residents tended to experience a variety of different traffic modes when they traveled for healthcare due to the factors including family income, geographical barriers, time, and type of diseases. The data about distance and time of 21930 routines among the streets and the hospitals in four traffic modes (driving mode, public traffic mode, bike-riding mode, and walking mode) were prepared by DataMap with the real-time data from amap.com. The distance was calculated according to the longitude and latitude data of streets and hospitals considering the traffic mode. Therefore, the distance between the same street and hospital was not the same in different traffic modes. The traffic time was estimated according to the distance and traffic mode. The distance and time data of routines are shown in Table 1.

The distribution of the streets and hospitals is shown in Figure 1.

2.2. Methods. The research process is shown in Figure 2.

2.2.1. Determination of the Size of Catchment Area. The core of 2SFCA and 3SFCA is to select the catchment area of public facilities to measure the traffic time threshold $\left(d_{0}\right)$. There have been many approaches for the determination of traffic time threshold [20, 21]. Mcgrail and Humphreys proposed that different threshold time can be determined according to the regional population density [22]. However, more recent studies stated that facilities with different sizes may have different levels of thresholds. Tao et al. point out that the larger the scale of facilities, the greater the attraction of facilities to demand points [23]. For example, 30 minutes is a common traffic time threshold $[5,24,25]$. Fu et al. set the maximum traffic time of the primary medical facilities to 20 minutes and the secondary medical facilities to 30 minutes, and for the tertiary medical facilities, it is available in the whole area [26]. Gao and $\mathrm{Li}$ set the time threshold of the secondary medical facilities to 30 minutes, the tertiary medical facilities to 45 minutes, and other facilities to 40 minutes [27].

Some studies also pointed out that traffic time threshold is also affected by traffic modes. Mao and Nekorchuk set the traffic time threshold to 30 minutes for the two traffic modes: by car or on feet combined with public bus [9]. Zhuge et al. found that when the traffic time is less than 30 minutes, residents tend to choose walking or riding bike; when the traffic time is more than 30 minutes and less than 60 minutes, residents choose both nonmotor vehicles and motor vehicles. When the traffic time is more than 60 minutes, residents tend to travel by motor vehicles [28].
Wang discussed the spatial distribution characteristics of accessibility under multilevel search radius and set different search radii in different traffic modes for different grades of hospitals. In the walking mode, the search radius of primary hospitals is between 15 minutes and 30 minutes, and in bikeriding, public traffic, and driving modes, the traffic time threshold of secondary hospitals is set to 30 minutes, 45 minutes, and 60 minutes [15].

Based on the findings above, the catchment in this study was divided into two subzones. The traffic time threshold of different types of medical facilities in different traffic modes was set as shown in Table 2.

2.2.2. Accessibility Measurement Method. The 3SFCA model proposed by Wan was adopted in this research, which is upgraded from the 2SFCA method. Given that people's needs for the health facilities are influenced by the availability of other health facilities nearby, this model deeply takes into account the competition effects among several service facilities within the scope of the service [6]. 3SFCA also assigns a competition weight to each medical facility based on the traffic time. Then, the competition weight is used for calculating the needs for service sites so as to minimize the overestimation.

Before the application of 3SFCA, the distance decay function $f(d)$ should be determined first. There are several distance decay functions shown in Table 3.

Actually, residents tend to choose healthcare resources that are closer to the place of residence (community and village), larger in scale and higher in level [29]. Such tendency in space can be similarly interpreted into a Gaussian function which gradually decreases with the increase of catchment area cost (time or distance). In recent years, Gaussian function-based methods as relatively well-developed methods were widely used in studies $[14,32,34,35]$. The Gaussian decay function without parameters was adopted in this study.

In this study, the 3SFCA method was implemented in the following steps.

Step 1. Calculating the catchment of the population $i$ according to the assigned medical facility $j$ in different traffic modes. Then, searching all medical facilities within the catchment area and assigning the Gaussian weight through equation (1) to each hospital according to the subarea where the hospital was located.

$$
G_{i j}=\frac{W_{i j}}{\sum_{k \in\left\{d_{i k}<d_{0}\right\}} W_{i k}},
$$

where $G_{i j}$ referred to the selecting weight between the population $i$ and the medical facility $j ; d_{i j}$ referred to the traveling cost per minute from population $i$ to any facility $k$ within the catchment area; $d_{0}$ referred to the travel time threshold measured by the size of catchment area; and $W_{i j}$ and $W_{i k}$ separately referred to the weight assigned to facilities $j$ and $k$. Therefore, equation (1) can also be expressed in the following way: 
TABLE 1: Distance and time of routines.

\begin{tabular}{lccccc}
\hline Routine ID & Street ID & Hospital ID & Distance (meters) & Time $(\mathrm{min})$ & Traffic mode \\
\hline 1 & D1 & H1 & 8659 & 27 & Driving \\
2 & D1 & H1 & 8648 & 62 & Public traffic \\
3 & D1 & H1 & 6905 & 92 & Walking \\
4 & D1 & H1 & 6786 & 27 & Bike-riding \\
$\ldots$ & $\ldots$ & $\ldots$ & $\ldots$ & $\ldots$ & $\ldots$ \\
21930 & D170 & H129 & 40058 & 160 & Bike-riding \\
\hline
\end{tabular}

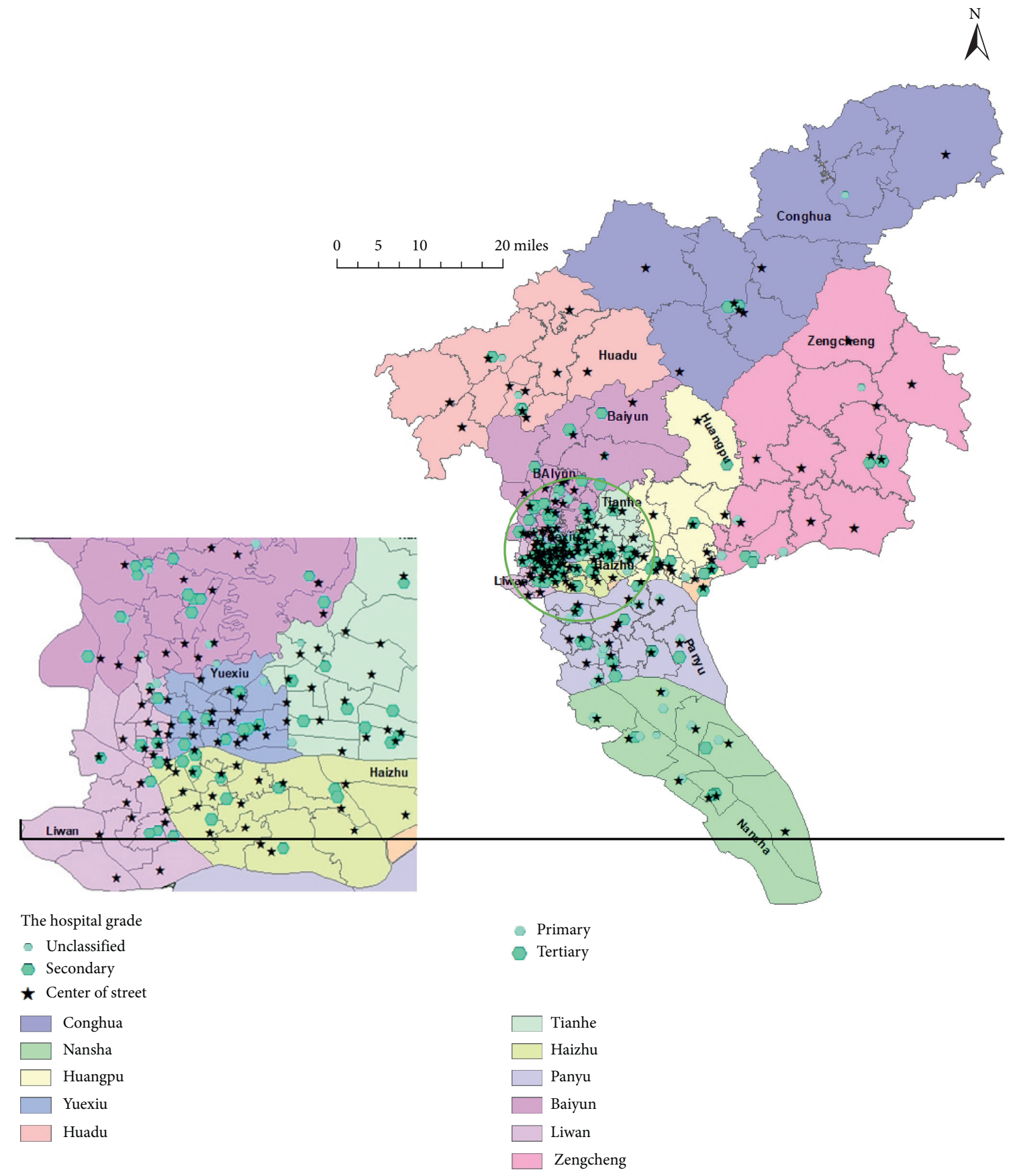

FIgURE 1: The spatial distribution of hospitals and streets. 


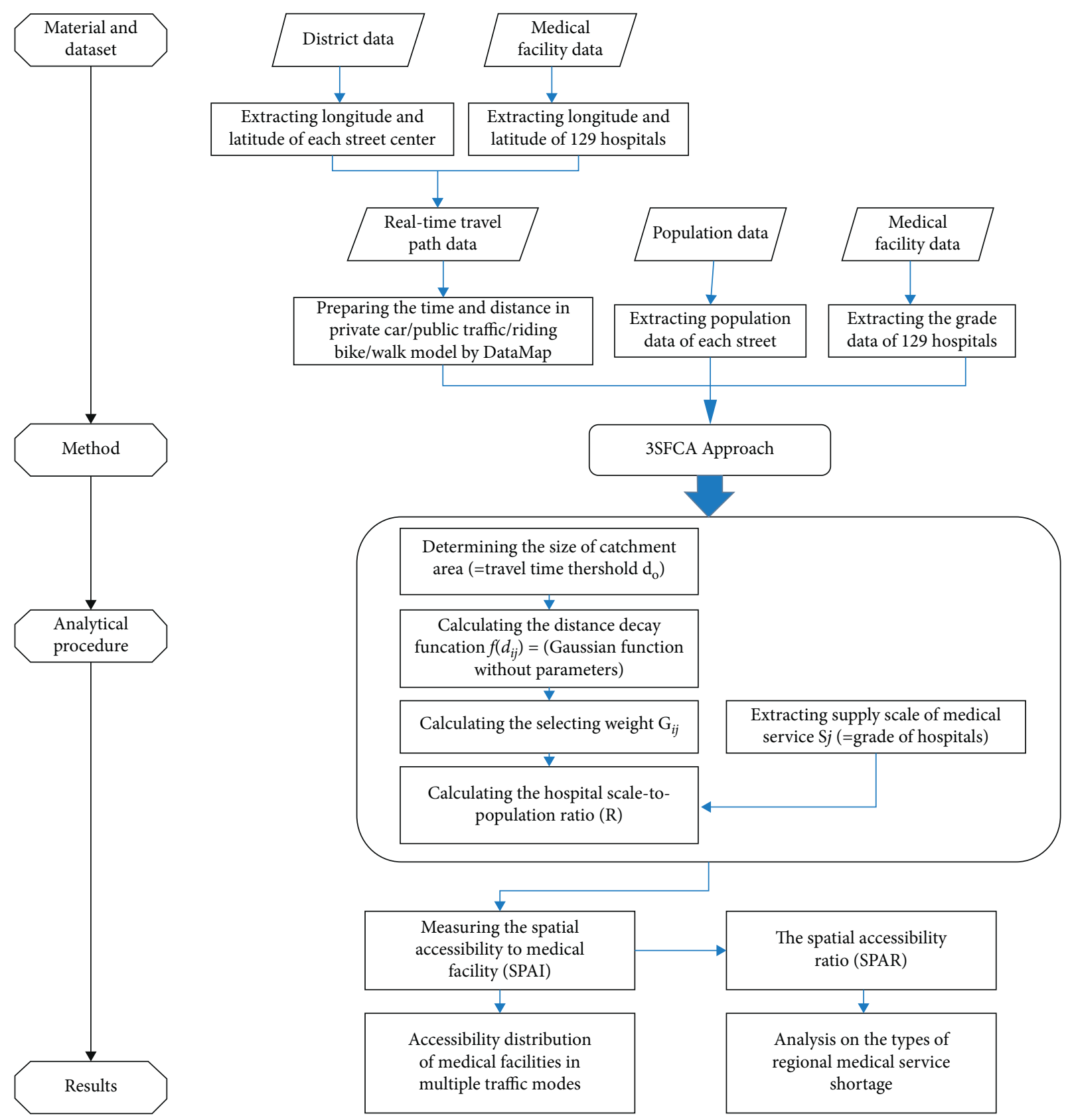

FIgURE 2: The research process of this study.

Table 2: Traffic time thresholds to medical facilities in different traffic Modes (in min).

\begin{tabular}{lcccc}
\hline Medical facility type & Driving & Public traffic & Bike-riding & Walking \\
\hline Third grade or above & 120 & 120 & 30 & 30 \\
Others & 60 & 60 & 15 & 15 \\
\hline
\end{tabular}

$$
G_{i j}=\frac{S_{j} f\left(d_{i j}\right)}{\sum_{k=1}^{n} S_{k} f\left(d_{i k}\right)}
$$

where $S_{j}$ referred to the supply scale of medical service of facility $j, d_{i j}$ was the travel time between $i$ and $j$, and $f\left(d_{i j}\right)$ was the Gaussian decay function without parameters $[33,34]$.
Step 2. Calculating the traffic time threshold for each medical facility $j$ to search all population within the catchment area. Then, calculating the physician-to-population ratio $(R)$ of facility $j$ in the following equation:

$$
R_{j}=\frac{S_{j}}{\sum_{r=1,2} \sum_{i \in D_{r}} G_{i j} P_{i} W_{r}},
$$


TABLE 3: Distance decay functions.

\begin{tabular}{lcc}
\hline Function name & Formula & Parameters \\
\hline Negative power function [29] & $f\left(d_{i j}\right)=d_{i j}^{-\beta}$ & $\beta$ \\
Negative exponential function [30] & $f\left(d_{i j}\right)=e^{-\beta d_{i j}}$ & $\beta$ \\
Gaussian function with parameters [6] & $f\left(d_{i j}\right)=e^{-\left(d_{i j} / \theta\right)^{2} / 2}$ & $\theta$ \\
Log-logistic function [31] & $f\left(d_{i j}\right)=1 / 1+\left(d_{i j} / \theta\right)^{\beta}$ & $\beta, \theta$ \\
Kernel density function [32] & $f\left(d_{i j}\right)=(3 / 4)\left(1-\left(d_{i j} / d_{0}\right)^{2}\right)$ & - \\
Gaussian function without parameters [33,34] & $f\left(d_{i j}\right)=\left(e^{-1 / 2\left(d_{i j} / d_{0}\right)^{2}}-e^{-1 / 2}\right) / 1-e^{-1 / 2}$ & - \\
\hline
\end{tabular}

where $W_{r}$ referred to the resistance of subzone $D_{r}$ and $P_{i}$ referred to the size of population $i$.

Step 3. Calculating the spatial accessibility of population $i$.

The traditional spatial accessibility was measured by equation (4) in which the impedance coefficient $\left(W_{r}\right)$ played an important role.

$$
\mathrm{SPAI}_{i}=\sum_{r=1,2} \sum_{j \in D_{r}} G_{i j} R_{j} W_{r} .
$$

In 3SFCA, it is assumed that local residents' needs for medical facilities nearby are influenced by the traffic cost of the residents going to this service site and the cost of residents who go to other health facilities nearby [6]. Actually, people's needs for the medical facility will be reduced when the facility nearby is available. The selecting weight $G_{i j}$ reflects the change. When there is only one medical facility near the population location, $G_{i j}$ is equal to 1 and will be decreased with the increase of number of available alternative facilities. The product of $G_{i j}, P_{i}$, and $W_{i j}$ refers to the adjusted potential needs of population $i$ for the facility $j$ after considering other competitive facilities.

Wan et al. pointed out that $W_{r}$ would result in the abnormal vibration of SPAI to influence the right measurement of accessibility. They proposed a new index, spatial accessibility rate (SPAR), to replace SPAI [6]. SPAR is calculated as the ratio of the SPAI of certain area to the average SPAI of all areas, as shown in the following equation:

$$
\operatorname{SPAR}_{i}=\frac{\mathrm{SPAI}_{i}}{\overline{\mathrm{SPAI}}}
$$

\section{Results and Discussion}

3.1. The Accessibility Distribution of Medical Facilities in Multiple Traffic Modes. As shown in Figure 3, the accessibility of medical facilities in the driving mode varies greatly. On the whole, the accessibility was lower in the center and higher in the surrounding areas. The reason was that the dense population had a relatively high demand for medical services in the central urban area with more medical facilities, while the population was sparse in the suburban areas. The driving model enabled urban residents in suburban areas to cross the geographical barriers to seek healthcare within the catchment area. However, the population in the central urban area was usually largest in a city, which meant that residents in this area were facing higher pressure for access to healthcare for a long time. Meanwhile, since local hospitals were incapable of meeting the oversaturated demand for medical services, the accessibility was usually lower than that in other areas.

The accessibility in public traffic mode was in average level and much lower than that in the driving mode. However, the distribution of accessibility in the investigated areas varied, while the accessibility in the central urban area was obviously higher than that in the suburban areas. Compared with the suburban area, the population density and medical facilities in the central urban area were disproportionally superior. Therefore, it was concluded that the advanced public catchment area network in the central downtown area guaranteed the residents to reach medical facilities within shorter time. However, taking comfort and convenience into consideration, the time people spent was longer and geographical barriers people needed to overcome were more complicated in the public traffic mode than those in the driving mode.

The accessibility in the bike-riding mode was tremendously lower than that in the driving mode and public traffic mode. The accessibility manifested a radial distribution in Yuexiu District and sporadic distribution in the suburban areas. The accessibility of Yuexiu District, the old urban areas, was better mainly because of the developed economy and abundant medical facilities. In the northern suburban areas, accessibility was much lower. Due to the scattered population and inadequate medical facilities, residents can hardly meet their healthcare needs in the bike-riding mode. In southern suburban areas like Panyu and Nansha, the accessibility was satisfactory since the healthcare needs of residents were mostly met.

The lowest accessibility of the investigated areas was in the walking mode. Generally, the accessibility increased from Yuexiu District as the center to the suburban areas. The larger the population was, the scarcer the medical facilities were. In Figure 3, some streets of Nansha and Zengcheng have higher accessibility than actual. This is the computational error incurred by the streets as the centroid of population is located too close to the hospitals when calculating the accessibility.

3.2. Spatial Accessibility Sensitivity Analysis in Multiple Traffic Modes. The spatial accessibility sensitivity was compared in different traffic modes with the SPAR index [13] . The description statistics of SPAR in different traffic modes is shown in Table 4.

According to the study of Wan [6], the SPAR index is divided into 6 ranks $(<0.5,0.51-1,1.01-1.5,1.51-2,2-2.5$, and $>2.5$ ). 
The result of accessibility distribution Private driving mode

SPAI
The result of accessibility distribution Public traffic mode

SPAI

\section{$\stackrel{N}{A}$}
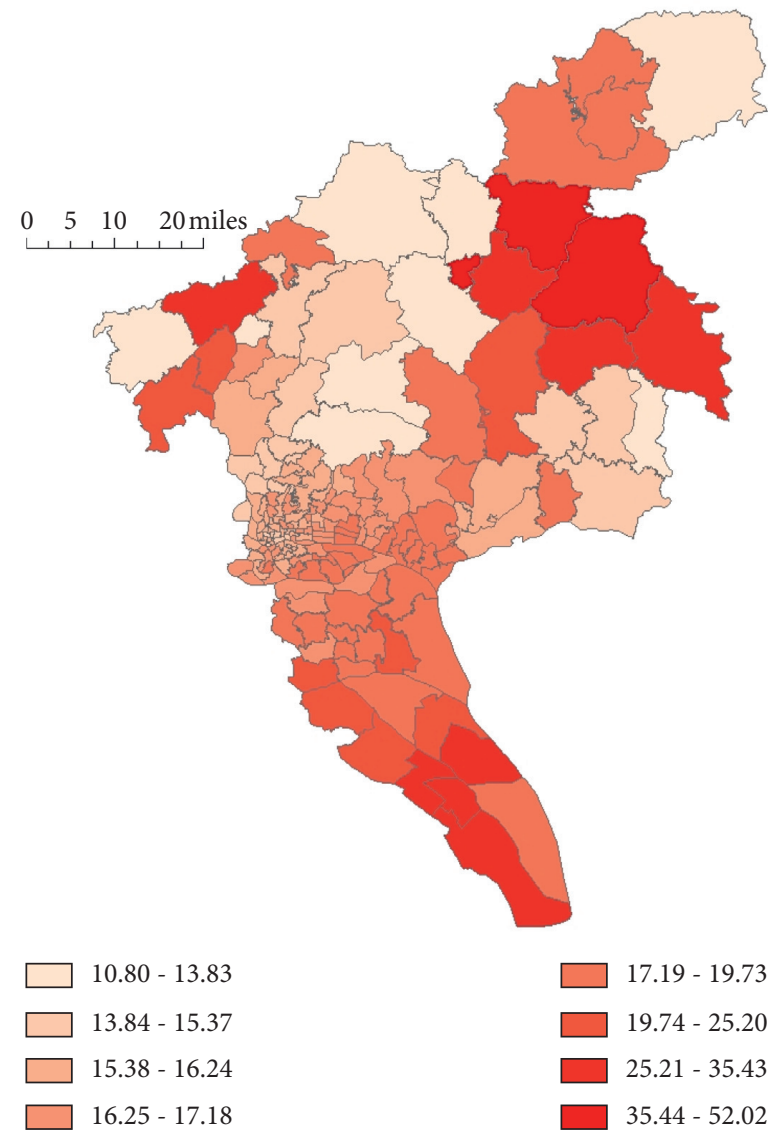

$\square \quad 17.19-19.73$
$\square \quad 19.74-25.20$
$\square \quad 25.21-35.43$
$\square \quad 35.44-52.02$

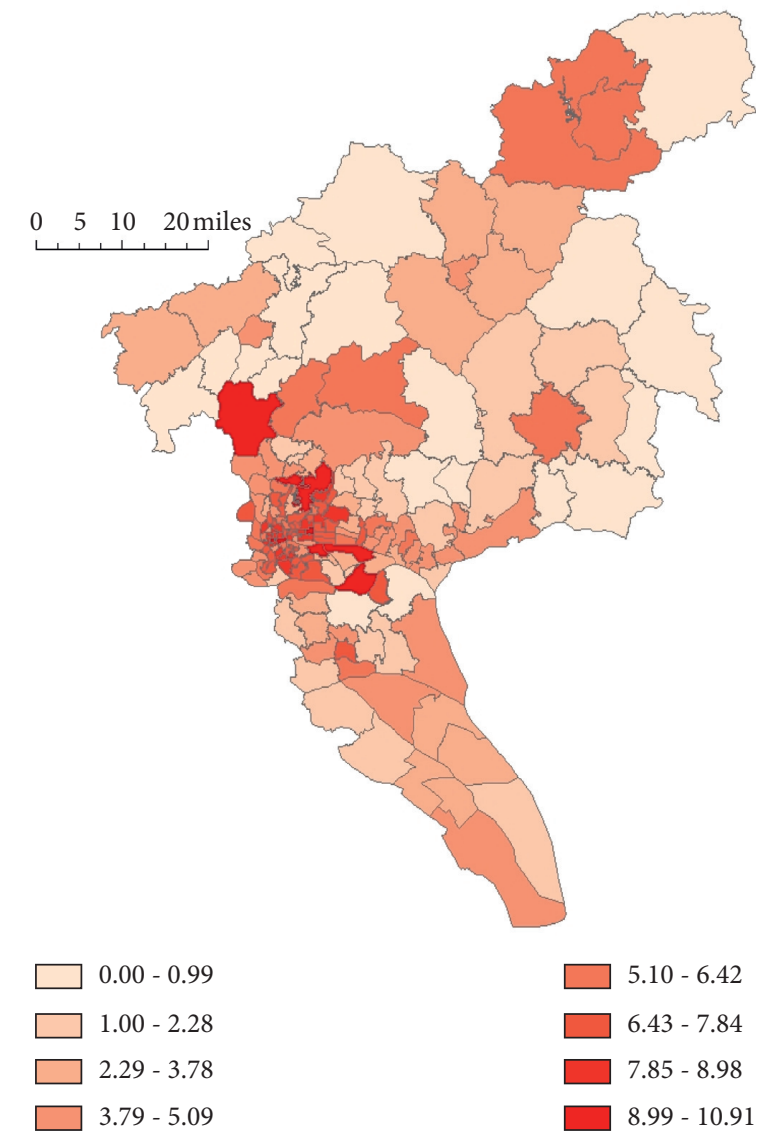

(a)

(b)

Figure 3: Continued. 
The result of accessibility distribution Bike-riding mode SPAI
The result of accessibility distribution Walking mode

SPAI
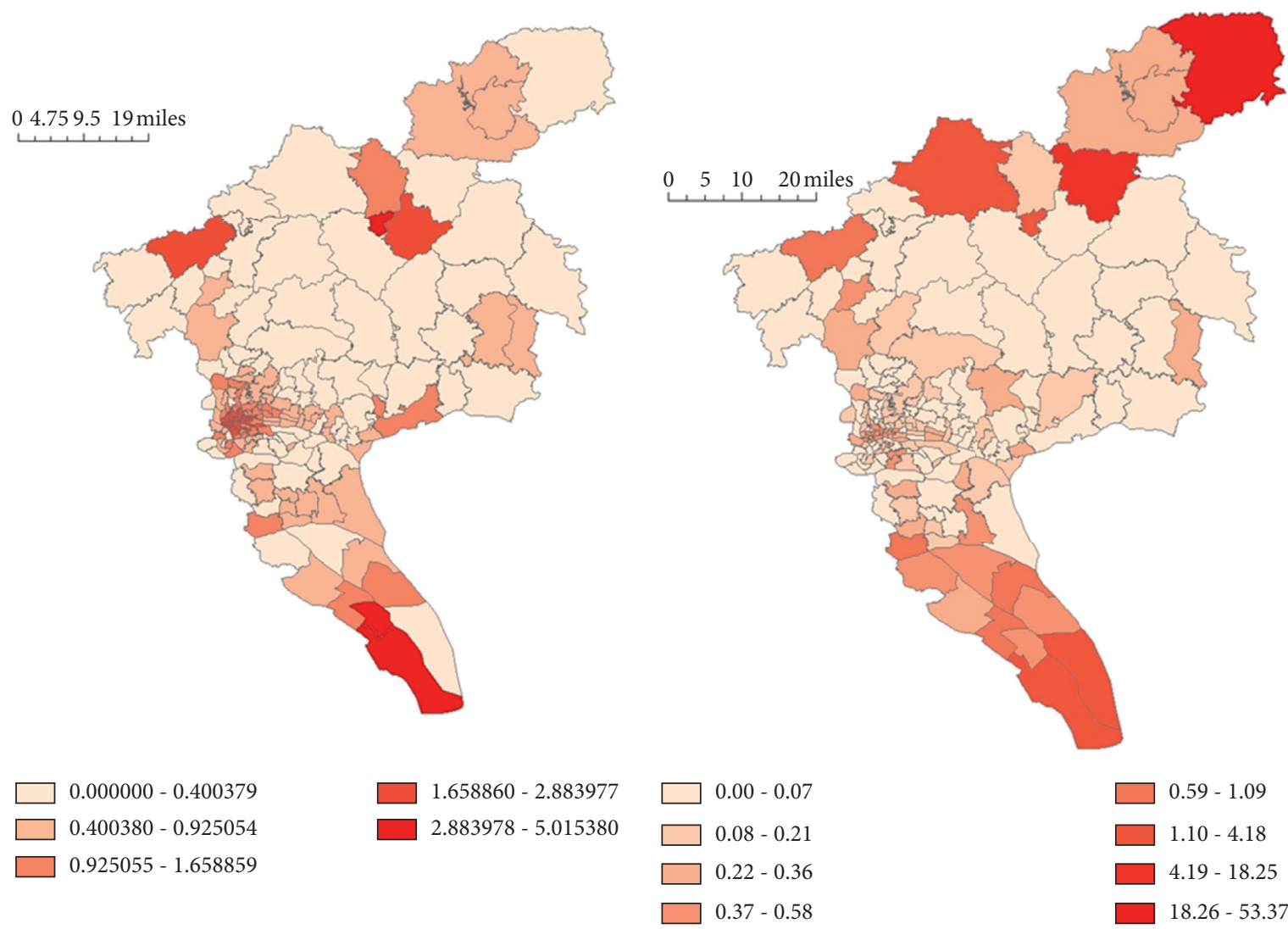

(c)

(d)

Figure 3: Accessibility distribution of medical facilities in multiple traffic modes.

TABLE 4: SPAR description statistics in different traffic modes.

\begin{tabular}{|c|c|c|c|c|c|}
\hline Traffic modes & Mean & Min & $\operatorname{Max}$ & Std. & $\sigma^{2}$ \\
\hline Private driving & 0.9827 & 0.6079 & 2.9276 & 0.3275 & 0.1072 \\
\hline Public traffic & 0.9827 & 0.0000 & 2.2170 & 0.5870 & 0.3446 \\
\hline Bike-riding & 0.9827 & 0.0000 & 5.7281 & 0.9618 & 0.9250 \\
\hline Walking & 0.9827 & 0.0000 & 83.3051 & 6.6946 & 44.8182 \\
\hline
\end{tabular}

Figure 4 shows the mapping results and the geographic distribution based on SPAR scores.

In the driving mode, the northeast suburban area had a higher score, while most areas with higher scores are mainly located in the center of the city in the public traffic mode. This hinted that in the suburban areas like the districts of Huadu, Conghua, Zengcheng, Panyu, and Nansha, according to the obtained distance time data, $94.7 \%$ people who lived in the streets of these areas had difficulties in taking bus to hospitals within the travel time threshold (SPAR $<0.9827$ ). This hinted that in the suburban areas, people had difficulties in taking bus to hospitals within the travel time threshold, while Yuexiu District as the center of the old downtown had the perfect public traffic facilities and routines, as well as more medical facilities. However, because of the fixed routines, travel by bus greatly restricted the traffic outside the center of the area and limited people to cross streets within the area. The situation of the bike-riding mode was similar to that of the walking mode. The streets with higher scores were mainly located in the southern and northern suburban areas of the city. In addition, in the bike-riding mode, the center of investigated area scored higher than that in the walking mode, and so did the travel time threshold of residents. This meant that residents were willing to move farther for better medical service in the bike-riding mode, which probably relieved 
The result of accessibility distribution Private driving mode

SPAI
The result of accessibility distribution Public traffic mode

SPAI

\section{$\stackrel{N}{N}$}

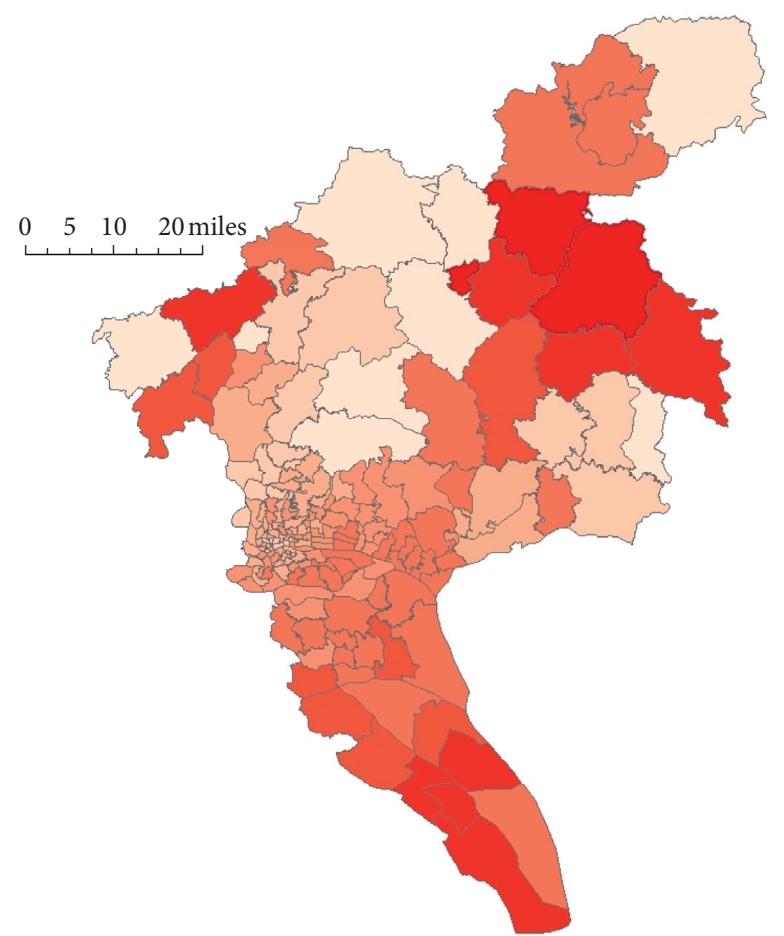

$10.80-13.83$

$13.84-15.37$

$15.38-16.24$

$16.25-17.18$

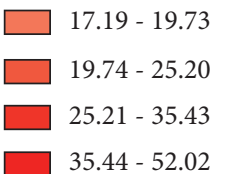

$35.44-52.02$

(a)

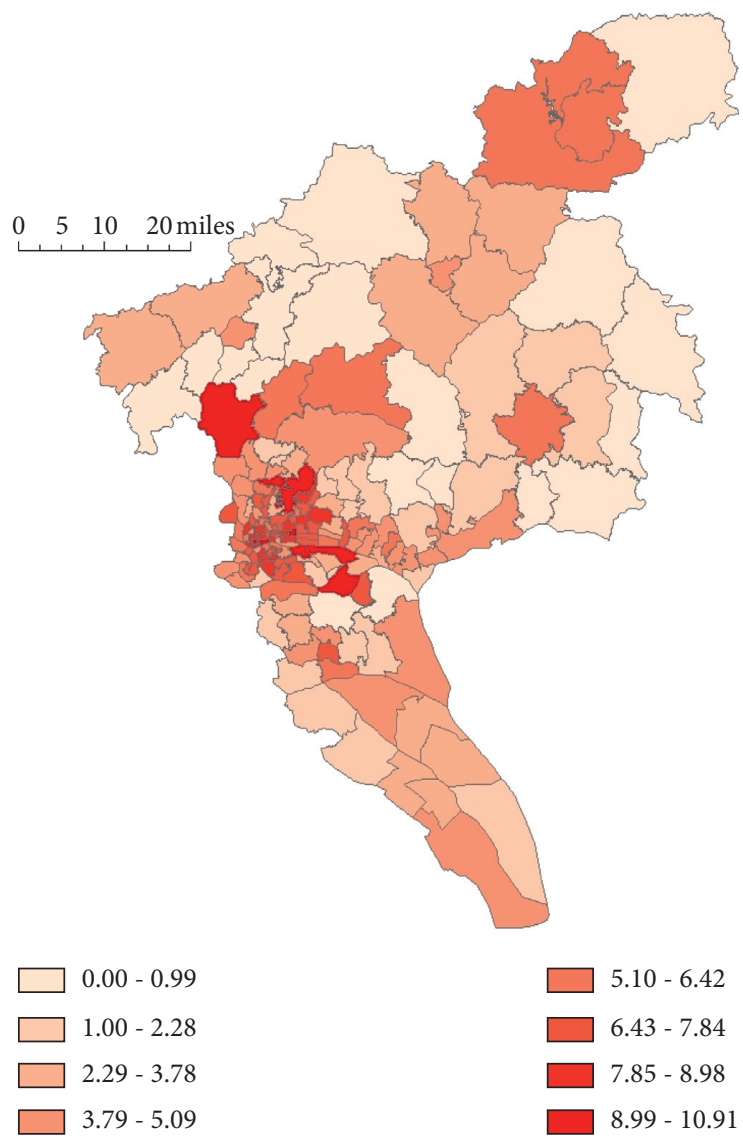

(b)

Figure 4: Continued. 
The result of accessibility distribution
Bike-riding mode SPAI
The result of accessibility distribution Walking mode

SPAI

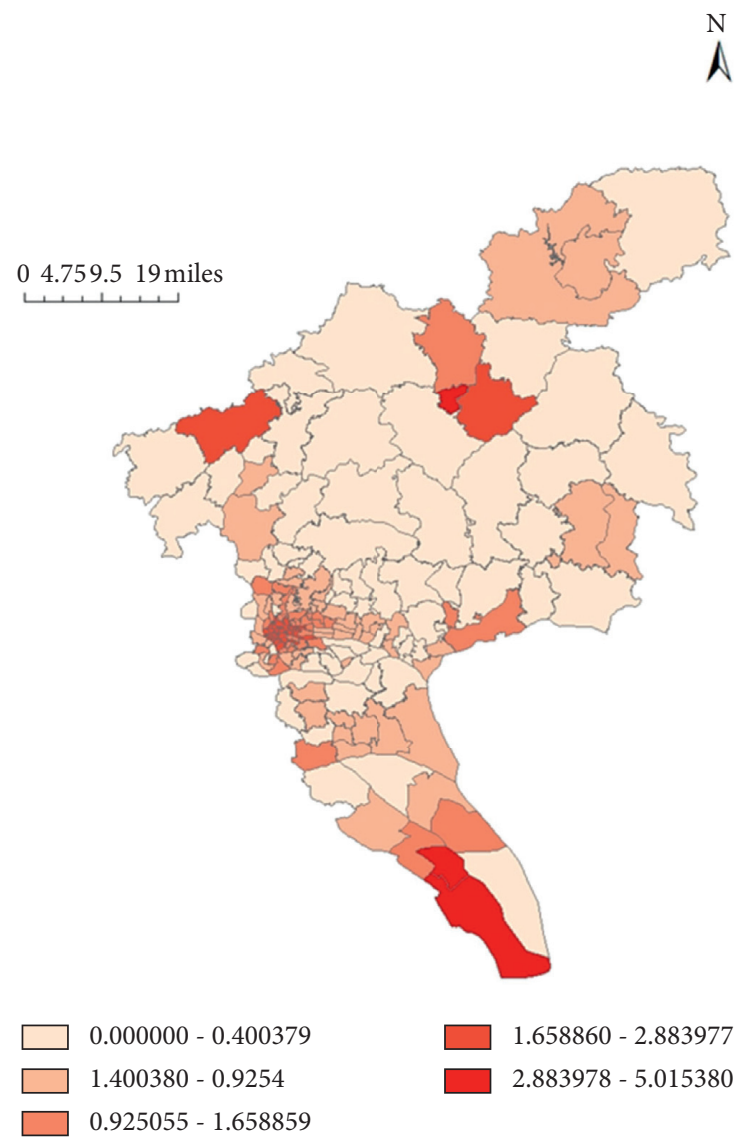

(c)

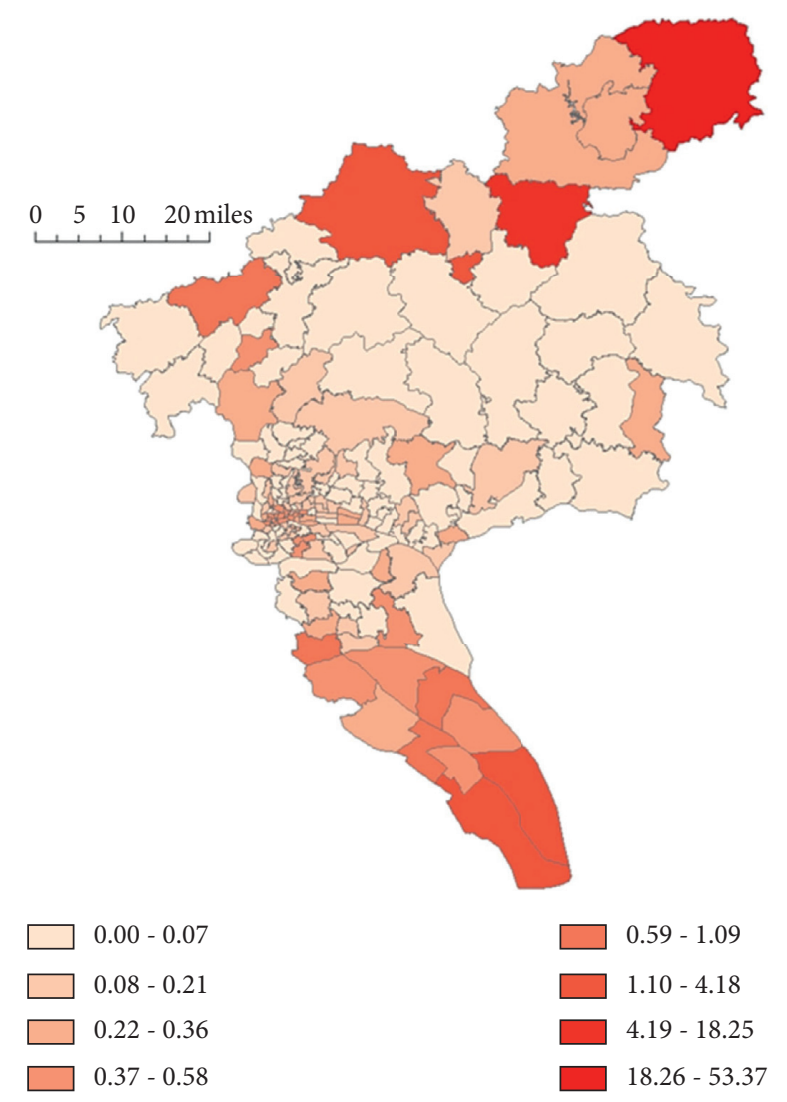

(d)

FIgURE 4: The distribution of SPAR of medical facilities in different traffic modes.

the crowdedness of medical service in the central downtown.

3.3. Analysis of the Types of Regions with Insufficient Medical Service. In terms of identifying the area with insufficient medical resources, Pathman and Ricketts took the composition of a region's residents, regional differences, and potential medical needs into consideration [36]. Zhu pointed out that the changes of low accessibility areas can be discovered in sensitivity analysis and that what medical services are scarce can be known by analyzing the causes for the lower spatial accessibility ratio in some areas [13]. In this study, the districts with severely insufficient medical service supply were divided into remote-area type (RAT) and local-inadequate-healthcare type (LIT) for further study.

As for RAT areas where the SPAR was less than 0.5 in the driving mode and public traffic mode and greater than 0.5 in the bike-riding and walking mode, since the residents were far away from the urban area, it was difficult for them to obtain high-quality medical service. For example, in Lvtian town located in Conghua District in the north of Guangzhou, it usually took residents at least 49.2 minutes to get to the nearest hospital. And the residents were also prevented from the fast and easy access to the nearest medical facilities by the poor public traffic. Therefore, the crucial means to enhance the accessibility of medical facilities in these areas relies on perfecting the traffic infrastructure to reduce the cost of residents' trip to medical facilities.

As for LIT areas where the SPAR was less than 0.5 in bike-riding and walking modes and greater than 0.5 in the driving mode and public traffic mode, people would drive or take bus to remote medical facilities rather than local ones. The scale or service level of medical facilities rather than traffic condition became the key factor for medical service. In this case, the average time residents spending on healthcare was extended and the accessibility of local medical facilities was decreased. Wang and Tang believed that remote areas often need additional medical supplies to compensate for their geographical location weakness compared to urban areas. At the same time, residents in some 
urban areas with high population density also need more supplies to meet the high healthcare demand [37].

As discussed above, in order to improve the accessibility of medical facilities of Guangzhou, the following measures are suggested.

For both sparsely populated surrounding areas and the crowded central urban areas, extra medical facilities are required to relieve the pressure of medical service. Apart from expanding the scale of hospitals and cultivating more high-capacity physicians, new facilities are needed to be set up according to the population distribution, traffic network, reconstruction of old districts, and other geographic factors.

In addition, for the remote areas with poor traffic condition, mobile medical service, online medical service, and regular circuit medical service should also be taken into account.

\section{Conclusions}

In this study, the 3SFCA method with Gaussian decay function was used to explore the accessibility of medical facilities in Guangzhou. We proposed to incorporate different traffic modes into the estimation of healthcare accessibility. We found that the traffic mode was an important factor to affect the ease of access to medical facilities, which should not be ignored in the accessibility measurement. Compared with the related methods $[6,8,9]$, the proposed multimode methods allowed a more realistic estimation of accessibility, which avoided the overestimation of the needs in gravity spatial model and the premature decay of distance decay functions such as negative exponential function and negative power function. This method provided a more reasonable model for the spatial access of basic medical services. The search radius varied according to the scale of different medical facilities, which smoothed the spatial layout for access to medical services.

In this study, the areas with low accessibility of medical facilities in Guangzhou were explored, which would help the authority to improve the layout of the medical facilities. However, the approach proposed in this study was also available for other cities, provided that the population census data and spatial data of medical facilities were prepared [32-34]. The analysis of the accessibility of medical facilities in Guangzhou would help to understand the imbalance of medical service and make better decisions on public healthcare supply.

It should be noted that the limitations of this study are as follows:

(i) With the data collected, the Gaussian function without parameters would result in the analysis with certain deviation. Notwithstanding its limitation, in future study, the approach will be improved by better decay functions with higherquality data about medical travels. In addition, more related factors including local economy, culture, residential conditions, medical service preference, and sociodemographic characteristics should also be considered. (ii) The difference of medical needs is ignored in this study. In fact, different communities have different medical needs. Women, children, and the elderly with higher demand for healthcare need more attention, especially in the areas with lower accessibility of medical facilities.

(iii) The investigation unit in this study is the streets which are the basic administrative organizations in China. The position of the street administrative office is taken as the centroid of population. In fact, the population is distributed in random. The size and the shape of the street area also varied. The estimation method of centroid of population should be improved for higher accuracy in the future study.

Besides all the above, this study mainly pays attention to the face-to-face medical service. In future study, the mobile medical service, online medical service, and regular circuit medical service as the complement medical services will be considered in the measurement of accessibility of medical facilities.

\section{Data Availability}

The data used to support the findings of this study are available from the first author upon request.

\section{Disclosure}

The funding sources had no role in the study design, data collection, analysis, interpretation, or the writing of this manuscript.

\section{Conflicts of Interest}

There are no conflicts of interest to declare.

\section{Acknowledgments}

This study was funded by the National Natural Science Foundation of China (71771059).

\section{References}

[1] N. Fullman, J. Yearwood, S. M. Abay et al., "Measuring performance on the healthcare access and quality index for 195 countries and territories and selected subnational locations: a systematic analysis from the global burden of disease study 2016," Lancet, vol. 391, pp. 2236-2271, 2018.

[2] Z. Chen and A. G.-O. Yeh, "Accessibility inequality and income disparity in urban China: a case study of Guangzhou," Annals of the American Association of Geographers, vol. 109, no. 1, pp. 121-141, 2019.

[3] T. S. Hare and H. R. Barcus, "Geographical accessibility and Kentucky's heart-related hospital services," Applied Geography, vol. 27, no. 3-4, pp. 181-205, 2007.

[4] M. R. Mcgrail and J. S. Humphreys, "Measuring spatial accessibility to primary care in rural areas: improving the effectiveness of the two-step floating catchment area method," Applied Geography, vol. 29, no. 4, pp. 533-541, 2009.

[5] W. Luo and F. Wang, "Measures of spatial accessibility to health care in a GIS environment: synthesis and a case study in 
the Chicago region," Environment and Planning B: Planning and Design, vol. 30, no. 6, pp. 865-884, 2003.

[6] N. Wan, B. Zou, and T. Sternberg, "A three-step floating catchment area method for analyzing spatial access to health services," International Journal of Geographical Information Science, vol. 26, no. 6, pp. 1073-1089, 2012.

[7] C. Wu, X. Ye, Q. Du, and P. Luo, "Spatial effects of accessibility to parks on housing prices in Shenzhen, China," Habitat International, vol. 63, pp. 45-54, 2017.

[8] X. Pan, M. P. Kwan, L. Yang, S. Zhou, Z. Zuo, and B. Wan, "Evaluating the accessibility of healthcare facilities using an integrated catchment area approach," International Journal Of Environmental Research And Public Health, vol. 15, no. 9, 2018.

[9] L. Mao and D. Nekorchuk, "Measuring spatial accessibility to healthcare for populations with multiple transportation modes," Health \& Place, vol. 24, pp. 115-122, 2013.

[10] X. Ma, F. Ren, Q. Du et al., "Incorporating multiple travel modes into a floating catchment area framework to analyse patterns of accessibility to hierarchical healthcare facilities," Journal of Transport \& Health, vol. 15, Article ID 100675, 2019.

[11] M. Langford, G. Higgs, and R. Fry, "Measuring access to primary health care using two-step floating catchment areas and a public/private multi-modal transport model," Plurimondi, vol. Sl, no. 16, 2017, http://plurimondi.poliba.it/index. $\mathrm{php} /$ Plurimondi/article/view/53.

[12] G. Higgs, R. Zahnow, J. Corcoran, M. Langford, and R. Fry, "Modelling spatial access to General Practitioner surgeries: does public transport availability matter?" Journal of Transport \& Health, vol. 6, pp. 143-154, 2017.

[13] M. Zhu, Measuring Primary Health Care Accessibility in Jianghan Plain: A Case Study of Xiantao, Central China Normal University, Wuhan, China, 2018.

[14] G. Chen, Research on the Spatio-Temporal Accessibility of Medical Service for the Elderly Using Ga2SFCA Method: A Case Study of Downtown Wuhan City, Central China Normal University, Wuhan, China, 2010.

[15] Q. Wang, Accessibility Analysis of Medical Service in Shanghai Based on Improved 2SFCA, Wuhan University, Wuhan, China, 2019.

[16] L. Che, "Using gravity accessibility model to evaluate the layout of shanghai's tertiary hospital," Chinese Journal of Health Statistics, vol. 30, no. 1, pp. 9-11, 2013.

[17] M. DiMarco, "Datamaps V0.50," 2020, http://datamaps. github.io..

[18] The People's Government of Guangzhou Municipality, "List of major hospitals in Guangzhou," 2020, http://www.gz.gov. cn/zwgk/zdly/ylwsjg/yljggk/content/mpost_3097589.html.

[19] National Health Commission of the People's Repulic of China, "Medical institions database," 2020, http://www.gov. $\mathrm{cn} /$ fuwu/bmfw/wjwyljgcx.

[20] D. P. Allan, "Catchments of general practice in different countries- a literature review," International Journal of Health Geographics, vol. 13, no. 1, p. 32, 2014.

[21] S. Mazumdar, X. Feng, P. Konings, I. McRae, and F. Girosi, “A brief report on primary care service area catchment geographies in New South Wales Australia," International Journal Of Health Geographics, vol. 13, no. 1, p. 38, 2014.

[22] M. R. McGrail and J. S. Humphreys, "Measuring spatial accessibility to primary health care services: utilising dynamic catchment sizes," Applied Geography, vol. 54, pp. 182-188, 2014.
[23] Z. Tao, Y. Cheng, and T. Dai, "Measuring spatial accessibility to residential care facilities in Beijing," Progress in Geography, vol. 33, no. 5, pp. 616-624, 2014.

[24] R. C. Lee, "Current approaches to shortage area designation," The Journal Of Rural Health, vol. 7, no. s4, pp. 437-450, 1991.

[25] L. Wang and D. Roisman, "Modeling spatial accessibility of immigrants to culturally diverse family physicians," The Professional Geographer, vol. 63, no. 1, pp. 73-91, 2011.

[26] L. Fu, Y. Wang, B. Zeng, Y. Mao, and M. Gao, "Spatial accessibility of medical facilities in Beibei District based on modified Two-step floating catchment area method," Journal of Geo-Information Science, vol. 21, no. 10, pp. 1565-1575, 2019.

[27] Y. Gao and M. Li, "Spatial accessibility of medical facilities based on modified two-step floating catchment area methodTake 9 districts in Shenyang as an example," Information Recording Materials, vol. 19, no. 2, pp. 115-118, 2018.

[28] C. Zhuge, C. Shao, X. Li, and M. Meng, "Commuter's choice behavior of travel time and travel mode," Journal of Transportation Systems Engineering and Information Technology, vol. 12, no. 2, pp. 126-131, 2012.

[29] P. Jia, I. M. Xierali, and F. Fahui Wang, "Evaluating and redemarcating the hospital service areas in Florida," Applied Geography, vol. 60, pp. 248-253, 2015.

[30] M. J. Hodgson, "An hierarchical location-allocation model for primary health care delivery in a developing area," Social Science \& Medicine, vol. 26, no. 1, pp. 153-161, 1988.

[31] J. J. D. Vries, P. Nijkamp, and P. Rietveld, "Exponential or power distance-decay for commuting? An alternative specification," Environment \& Planning A, vol. 41, no. 2, pp. 461-480, 2009.

[32] D. Dai and F. Wang, "Geographic disparities in accessibility to food stores in southwest Mississippi," Environment and Planning B: Planning and Design, vol. 38, no. 4, pp. 659-677, 2011.

[33] D. Dai, "Racial/ethnic and socioeconomic disparities in urban green space accessibility: where to intervene?" Landscape and Urban Planning, vol. 102, no. 4, pp. 234-244, 2011.

[34] D. Dai, "Black residential segregation, disparities in spatial access to health care facilities, and late-stage breast cancer diagnosis in metropolitan Detroit," Health \& Place, vol. 16, no. 5, pp. 1038-1052, 2010.

[35] F. Zhang, D. Li, S. Ahrentzen, and J. Zhang, "Assessing spatial disparities of accessibility to community-based service resources for Chinese older adults based on travel behavior: a city-wide study of Nanjing, China," Habitat International, vol. 88, Article ID 101984, 2019.

[36] D. E. Pathman and T. C. Ricketts, "When access-to-care indicators meet: designated shortage areas and avoidable hospitalizations," Archives of Family Medicine, vol. 8, no. 6, pp. 492-493, 1999.

[37] M. F. Guagliardo, "Spatial accessibility of primary care: concepts, methods and challenges," International Journal of Health Geographics, vol. 3, no. 3, pp. 101-112, 2004. 